\title{
Effect of Preceding Crop, Rates and Splitting of Nitrogen Fertilizer on Bread Wheat Production and Nitrogen Use Efficiency \\ Ayat B. H. Gad ${ }^{1}$; E. M. M. Shalaby ${ }^{1}$; H. G. Hassanein ${ }^{2}$; E. A. Ali ${ }^{1}$ and M. T. Said ${ }^{1}$ \\ ${ }^{1}$ Agronomy Dept., Fac. Agric., Assiut Univ., Egypt. \\ ${ }^{2}$ Soils and water Dept., Fac. Agric., Assiut Univ., Egypt.
}

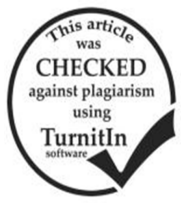

\section{ABSTRACT}

A field experiment was conducted at the Agronomy Department Farm, Faculty of Agriculture, Assiut University during 2015/2016 and 2016/2017 seasons to study the effect of preceding crop, rates and splitting of nitrogen fertilizer on bread wheat production and nitrogen use efficiency. The experiment was carried out in randomization complete block design (RCBD) using splitsplit plot arrangement with three replications. Cropping system (corn-clover-wheat and corn-fallow-wheat) were assigned in main-plots while nitrogen fertilizer rates $(56.25,75.00$ and $93.75 \mathrm{~kg} \mathrm{~N} / \mathrm{fed})$ were allotted in sub-plots and splitting doses of nitrogen (two, three and four equal doses) were allocated in sub-sub plots. The obtained data showed that plant height, spike length, number of spikes $/ \mathrm{m}^{2}$, number of grains/spike, weight of grains/spike, seed index, grain yield, straw yield and nitrogen use efficiency were significantly affected by cropping sequence in favor of planting wheat after clover (fahl berseem) in the two growing seasons except nitrogen use efficiency in the first season. Increasing nitrogen fertilizer rates resulted in significant increase in all previous traits except nitrogen use efficiency trait which was decreased by increasing nitrogen fertilizer rates in both seasons. Moreover, splitting nitrogen rates into four equal doses significantly increased all studied traits as compared to three or two equal splits in the two growing seasons. Also, all interactions had a significant effect on all studied traits in both seasons. The highest mean values of grain yield (27.47 and 28.22 ard/fed in the first and second seasons, respectively) were obtained from the sequence of corn-clover-wheat when received highest nitrogen rate $(93.75 \mathrm{~kg}$ $\mathrm{N} / \mathrm{fed}$ ) which was applied at four equal doses.

Keywords: Bread wheat, Preceding crop, Nitrogen fertilizer rates and splitting number.

\section{INTRODUCTION}

Wheat crop (Triticum sp. L.) considers the most important cereal crop in the world with regard to cultivated area and production. The cultivated area of wheat in Egypt during 2018 season was about 1.32 million hectare with the total yield production of 8.45 metric ton (USDA, 2018). While the total consumption reached about 19.6 million metric ton. So, increasing wheat production in order to reduce the gap between production and consumption are the strategic aim. The previous aim could be occur by increasing soil fertility via legume crops or mineral fertilization.

Crop rotations avoid yield depressions under monoculture which increase populations of microorganisms that are pathogenic and decrease population of antagonistic microorganisms in the crop root rhizosphere (Cook, 1984) and reduce production of phytoxic allelopathic chemicals and improve physical and chemical conditions of soil. Dogan and Bilgili (2010) indicated that both the previous crop and $\mathrm{N}$ fertilization treatments significantly affected all components of the winter wheat cultivars. There was a close relationship between $\mathrm{N}$ fertilization and yield components. In generally, a previous crop of alfalfa gave the highest value of all components. Hefny (2012) cleared that there were significant differences between crop sequences with wheat for plant height, number of spikes $/ \mathrm{m}^{2}$, number of grains/spike, grains weight/spike, 1000-grain weight, grain yield, straw and biological yields/fed. Zaheer et al. (2015) noticed that plots grown with mungbean (sole) had higher $\mathrm{N}$ content at both occasions (before sowing $0.41 \mathrm{~g} \mathrm{~kg}^{-1}$ and after harvest $0.38 \mathrm{~g} \mathrm{~kg}^{-1}$ ) as compared with other cropping patterns. All parameters were significantly affected by cropping pattern and nitrogen application. Mungbean (sole) or intercropped with groundnut as preceding crop had significantly produced taller plants, more: grains spike $^{-1}$, thousand grain weight, biological yield and grain yield.

Nitrogen is an essential nutrient for growth, development and biomass production. It also plays important roles in physiological functions (Kaur et al. 2016). El-Temsah (2017) revealed that yield components of wheat were significantly affected by nitrogen rates.
Plant height, spikes number $/ \mathrm{m}^{2}$, main spike length, main spike weight and grains number per main spike of wheat were markedly varied being in increasing order with different nitrogen rates 60,80 and $100 \mathrm{~kg} \mathrm{~N} / \mathrm{fad}$. The application of $100 \mathrm{~kg} \mathrm{~N} /$ fad scored the maximum values of plant height $(118.83 \mathrm{~cm})$, spikes number $/ \mathrm{m}^{2}(655.17)$, main spike length $(13.09 \mathrm{~cm})$, main spike weight $(5.54 \mathrm{~g})$ and grains numbers per main spike (69.33). Haque et al. (2017) reported that different $\mathrm{N}$ levels had a significant effect on the spike length, number of grains/spike, thousand grain weight, grain yield, straw yield and nitrogen use efficiency.

Due to nitrogen loss through gaseous plant emission, soil denitrification, volatilization and leaching, $\mathrm{N}$ use efficiency for cereal production is low (33\%) throughout the world. Usman and Ur-Rehman (2014) concluded that grain yield and yield components were significantly affected by $\mathrm{N}$ fertilizer timing and splitting. Application of nitrogen in three equal splits $(50 \mathrm{~kg} \mathrm{~N}$ at sowing $+50 \mathrm{~kg} \mathrm{~N}$ at first irrigation $+50 \mathrm{~kg} \mathrm{~N} / \mathrm{ha}$ at second irrigation) produced highest spike length $(9.3 \mathrm{~cm})$, spikes number $/ \mathrm{m}^{2}$ (314), grains number/spike (51.9), 1000-grain weight (41.6 g) and grain yield $\left(6250 \mathrm{~kg} \mathrm{ha}^{-1}\right)$. Singh et al. (2016) stated that the use of three split $\mathrm{N}$ applications increased all growth and yield attributes. The best $\mathrm{N}$ split strategy corresponded to three $\mathrm{N}$ splits: at planting, crown root initiation (CRI), and panicle initiation (PI) stages.

The objective of this study is to investigate the effect of preceding crop, rates and splitting of nitrogen fertilizer on bread wheat production and nitrogen use efficiency.

\section{MATERIALS AND METHODS}

This investigation was carried out at Agronomy Department Farm, Faculty of Agriculture, Assiut University, during 2015/2016 and 2016/2017 seasons to study the effect of preceding crop, rates and splitting of nitrogen fertilizer on bread wheat (Sids-12 cultivar) production and nitrogen use efficiency.

The experiment was carried out in randomization complete block design (RCBD) using split-split plot arrangement with three replications. The cropping system 
(corn-clover-wheat and corn-fallow-wheat) were a signed in the main-plots while the nitrogen fertilizer rates $(56.25,75$ and $93.75 \mathrm{~kg} \mathrm{~N} /$ fed: application of 75,100 and $125 \%$ of the recommended $\mathrm{N}$ fertilizer rate/fed, respectively) were distributed in the sub-plots and different split doses (two, three and four equal doses) were arranged in the sub-sub plots. The experimental unit area was $10.5 \mathrm{~m}^{2}(3 \times 3.5)$ in each season. The clover (fahl berseem) was planted on September $22^{\text {nd }}$ in both seasons. After cutting the clover crop on December $6^{\text {th }}$ in both seasons then, wheat was sown on December $18^{\text {th }}$ and $19^{\text {th }}$ in $2015 / 2016$ and $2016 / 2017$ seasons, respectively using broadcasting (conventional tilled flat method).

The nitrogen fertilization rates were applied in the form of urea $(46.5 \% \mathrm{~N})$ as a source of nitrogen.

The third variable was the splitting of nitrogen fertilization doses. The treatments in this respect were as follows:

Table 2. Some chemical properties of experimental site

\begin{tabular}{|c|c|c|c|c|c|c|c|c|}
\hline \multirow{3}{*}{ Type of soil } & \multicolumn{4}{|c|}{ 2015/2016 } & \multicolumn{4}{|c|}{ 2016/2017 } \\
\hline & \multirow{2}{*}{$\begin{array}{c}\text { Organic } \\
\text { matter \% }\end{array}$} & \multicolumn{3}{|c|}{ Available elements per $\mathrm{mg} / \mathrm{kg}$ soil } & \multirow{2}{*}{$\begin{array}{c}\text { Organic } \\
\text { matter \% }\end{array}$} & \multicolumn{3}{|c|}{ Available elements per $\mathrm{mg} / \mathrm{kg}$ soil } \\
\hline & & $\mathbf{N}$ & $\mathbf{P}$ & $\mathbf{K}$ & & $\mathbf{N}$ & $\mathbf{P}$ & $\mathbf{K}$ \\
\hline Before cultivation & 1.59 & 70.00 & 89.60 & 880.00 & 1.42 & 82.00 & 96.00 & 780.00 \\
\hline After clover & 1.81 & 111.70 & 106.70 & 680.00 & 1.94 & 140.90 & 146.00 & 860.00 \\
\hline After fallow-wheat & 1.28 & 100.00 & 84.45 & 540.00 & 1.61 & 107.80 & 76.55 & 780.00 \\
\hline After clover-wheat & 1.59 & 105.80 & 86.25 & 720.00 & 2.27 & 103.00 & 169.40 & 920.00 \\
\hline
\end{tabular}

The above analysis was carried out in the Agricultural Research Center Soil, Water \& Environment Research. Institute Unit of Analysis \& Studies, Faculty of Agriculture, Assiut University.

At harvest, number of spikes $/ \mathrm{m}^{2}$ for each experimental unit was recorded then ten guarded stems were taken randomly from each experimental unit and the follow traits were measured i.e. plant height $(\mathrm{cm})$, spike length $(\mathrm{cm})$, grains number/spike, grains weight/spike $(\mathrm{g})$ and seed index (g).

Grain yield/fed: All harvested plants for each experimental unit were threshed then grain weighted and transferred into $\mathrm{ardab} / \mathrm{fed}\left(\mathrm{ardab}=150 \mathrm{~kg}\right.$, fed $\left.=4200 \mathrm{~m}^{2}\right)$.

Straw yield for each plot was weighted then transferred into ton/fed.

Nitrogen Use Efficiency (NUE): The nitrogen use efficiency was judged by the quantity of grain yield produced per $\mathrm{kg} \mathrm{N}$ applied (kg grains/kg N) according to Ali (2011).

\section{Statistical analysis:}

Using CoStat (Version 6.303, CoHort, USA, 1998-2004). The significant means of any trait studied were compared using LSR at 5\% probability level according to Gomez and Gomez (1984).

\section{RESULTS AND DISCUSSION}

\section{Yield attributes:}

The results demonstrated in Tables 3 to 8 indicate that plant height, spike length, spikes number $/ \mathrm{m}^{2}$, grains number/spike, grains weight/spike and seed index traits were affected significantly by cropping sequence in both seasons. In generally, planting wheat after a legume crop produced the maximum yield and its components as compared with planting wheat after fallow. The sequence of corn-clover-wheat produced the highest mean value of mentioned traits $(105.10 \mathrm{~cm}, 11.84 \mathrm{~cm}, 412.59,75.66,3.29$ $\mathrm{g}$ and $48.51 \mathrm{~g}$ of plant height, spike length, spikes
1. Splitting of nitrogen fertilization doses into two equal parts before the second and third irrigations $\left(\mathrm{Sp}_{2}\right)$.

2. Splitting of nitrogen fertilization doses into three equal parts before the second, the third and the fourth irrigation $\left(\mathrm{Sp}_{3}\right)$.

3. Splitting of nitrogen fertilization doses into four equal parts before the second, third, fourth and fifth irrigations $\left(\mathrm{Sp}_{4}\right)$.

The soil used for these experiments was clay and its structure as presented in Table 1.

Table 1. Some mechanical properties of experimental site

\begin{tabular}{lccc}
\hline \multicolumn{3}{c}{ Particle size distribution (\%) } & \multirow{2}{*}{ Soil Texture } \\
\cline { 1 - 2 } Sand & Silt & Clay & \\
\hline 8.64 & 28.00 & 63.36 & Clay \\
\hline
\end{tabular}

number $/ \mathrm{m}^{2}$, grains number/spike, grains weight/spike and seed index in first season, respectively being $111.88 \mathrm{~cm}$, $12.70 \mathrm{~cm}, 454.37,67.13,3.01 \mathrm{~g}$ and $46.84 \mathrm{~g}$ in the second season in the same order). This trend could be ascribed to the legume ability of fix atmospheric $\mathrm{N}_{2}$, thereby increasing soil $\mathrm{N}$ as indicated in Table 2. This in turn may enhance the photosynthesis process and consequently increasing the metabolites synthesized in wheat plant. Similar results were obtained by Said (2007), Dogan and Bilgili (2010), Hefny (2012) and Zaheer et al. (2015).

Data presented in the same Tables reveal that nitrogen fertilizer rates had a significant influence on above mentioned traits in the both seasons. Maximum mean values of plant height, spike length, spikes number $/ \mathrm{m}^{2}$, grains number/spike, grains weight/spike and seed index were noted when $93.75 \mathrm{~kg} \mathrm{~N} /$ fed was applied in both seasons. The increased in the plant height and spike length due to $\mathrm{N}$ fertilizer could be ascribed to the role of $\mathrm{N}$ in stimulating cell division and elongation, consequently internodes elongation in addition and development. Also, it is clear from these data that $\mathrm{N}$ fertilization enhanced the vegetative growth of the plants; increased photosynthetic activity and the metabolites required to produce vigorous growth and consequently produced maximum yield and its components. These results are harmony with those obtained by Dogan and Bilgili (2010), Abedi et al. (2011), El-Agrodi et al. (2011), Mandic et al. (2015), Zaheer et al. (2015), Mansour et al. (2016), ElTemsah (2017) and Haque et al. (2017).

Here too, the illustrated data focus that the above mentioned traits reacted significantly to split nitrogen fertilizer in both seasons. The highest mean values of these traits $(102.49 \mathrm{~cm}, 11.74 \mathrm{~cm}, 425.78,76.55,3.31 \mathrm{~g}$ and 47.04 $\mathrm{g}$ of plant height, spike length, spikes number $/ \mathrm{m}^{2}$, grains number/spike, grains weight/spike and seed index in first season, respectively being $108.00 \mathrm{~cm}, 12.98 \mathrm{~cm}, 477.33$, $69.25,3.19 \mathrm{~g}$ and $47.26 \mathrm{~g}$ in the second season in the same 
order) were recorded when $\mathrm{N}$ fertilizer was applied in four equal doses before the second, third, fourth and fifth irrigations. Otherwise, the lowest mean values of these characters were obtained when $\mathrm{N}$ fertilizer was applied in two equal doses before the second and third irrigations in both seasons. The present trend could be ascribed to the splitting nitrogen fertilizer may decrease the rate of leaching and splitting the fertilizers at different stages of growth is very important to face the different requirements of nitrogen. This could maximize the benefit of $\mathrm{N}$. These results are in line with those obtained by Abedi et al. (2011), El-Agrodi et al. (2011), Ercoli et al. (2013), Usman and Ur-Rehman (2014) and Singh et al. (2016).

Furthermore, the first order interaction of cropping sequence $\times \mathrm{N}$ rates proved to be significant on plant height, spike length, spikes number $/ \mathrm{m}^{2}$, grains number/spike, grains weight/spike and seed index in both seasons. The highest mean values of these traits were obtained from the sequence of corn-clover-wheat with $93.75 \mathrm{~kg} \mathrm{~N} / \mathrm{fed}$ in both seasons. Here too, the first order interaction of cropping sequence $\times$ splitting number turned to be significant in this respect in both seasons. The highest mean values of these traits were obtained from the sequence of corn-clover-wheat with four equal doses of $\mathrm{N}$ application in both seasons. Also, the first order interaction of $\mathrm{N}$ rates $\times$ splitting number proved to be significant in this respect in both seasons. The highest mean values of these traits were obtained from wheat plants fertilized by $93.75 \mathrm{~kg} \mathrm{~N} /$ fed when it applied at four equal doses in both seasons. In addition, the second order interaction was significant in this respect in both seasons.

Table 3. Effect of crop sequence, $N$ rates, splitting and their interactions involved on plant height $(\mathrm{cm})$.

\begin{tabular}{|c|c|c|c|c|c|c|c|c|c|c|c|c|c|}
\hline \multirow{2}{*}{ Seasons } & \multirow{2}{*}{$\begin{array}{c}\text { cropping } \\
\text { sequence } \\
\text { N rates (N) } \\
\text { Splitting (Sp) }\end{array}$} & \multicolumn{3}{|c|}{ Corn-Clover-Wheat } & \multirow{2}{*}{ Mean } & \multicolumn{3}{|c|}{ Corn-Fallow-Wheat } & \multirow{2}{*}{ Mean } & \multicolumn{3}{|c|}{$\mathbf{N} \times \mathbf{S P}$} & \multirow{2}{*}{ Mean } \\
\hline & & $\mathbf{N}_{1}$ & $\mathbf{N}_{2}$ & $\mathbf{N}_{3}$ & & $\mathbf{N}_{1}$ & $\mathbf{N}_{2}$ & $\mathbf{N}_{3}$ & & $\mathbf{N}_{1}$ & $\mathbf{N}_{2}$ & $\mathbf{N}_{3}$ & \\
\hline \multirow{3}{*}{ 2015-16 } & $\mathrm{Sp}_{2}$ & $102.10^{\mathrm{F}}$ & $102.60^{\mathrm{EF}}$ & $103.70^{\mathrm{DE}}$ & $102.80^{\mathrm{c}}$ & $95.67^{\mathrm{J}}$ & $96.27^{\mathrm{IJ}}$ & $97.03^{\mathrm{HI}}$ & $96.32^{\mathrm{e}}$ & $98.88^{\mathrm{e}}$ & $99.43^{\mathrm{e}}$ & $100.37^{\mathrm{d}}$ & $99.56^{C}$ \\
\hline & $\mathrm{Sp}_{3}$ & $104.47^{\mathrm{CD}}$ & $105.47^{\mathrm{BC}}$ & $106.67^{\mathrm{AB}}$ & $105.53^{\underline{\mathrm{b}}}$ & $96.80^{\mathrm{H}-\mathrm{J}}$ & $97.10^{\mathrm{HI}}$ & $98.17^{\mathrm{GH}}$ & $97.36^{\mathrm{d}}$ & $100.63^{\mathrm{d}}$ & $101.28^{\mathrm{cd}}$ & $102.42^{\mathrm{ab}}$ & $101.44^{B}$ \\
\hline & $\mathrm{Sp}_{4}$ & $106.33^{\mathrm{B}}$ & $106.80^{\mathrm{AB}}$ & $107.80^{\mathrm{A}}$ & $106.98^{\mathrm{a}}$ & $97.53^{\mathrm{G}-\mathrm{I}}$ & $97.77^{\mathrm{GH}}$ & $98.70^{\mathrm{G}}$ & $98.00^{\mathrm{d}}$ & $101.93^{\mathrm{bc}}$ & $102.28^{\mathrm{b}}$ & $103.25^{\mathrm{a}}$ & $102.49^{A}$ \\
\hline \multirow[t]{2}{*}{ Mean } & & $104.30^{\mathrm{C}}$ & $104.96^{\underline{B}}$ & $106.06^{\mathrm{A}}$ & 105.10 & $96.67^{\mathrm{E}}$ & $97.04^{\mathrm{E}}$ & $97.97^{\mathrm{D}}$ & 97.23 & $100.48^{\epsilon}$ & $101.00^{\mathrm{B}}$ & $102.01^{\mathrm{A}}$ & -- \\
\hline & $\mathrm{Sp}_{2}$ & $106.00^{\mathrm{C}}$ & $108.40^{\mathrm{C}}$ & $112.67^{\mathrm{B}}$ & $109.02^{\mathrm{c}}$ & $91.50^{\mathrm{H}}$ & $98.40^{\mathrm{F}}$ & $98.73^{\mathrm{F}}$ & $96.21^{\mathrm{f}}$ & $98.75^{\mathrm{g}}$ & $103.40^{\mathrm{ef}}$ & $105.70^{\mathrm{d}}$ & $102.62^{C}$ \\
\hline \multirow[t]{2}{*}{ 2016-17 } & $\mathrm{Sp}_{3}$ & $7.77^{\mathrm{C}}$ & $113.37^{\mathrm{B}}$ & $114.17^{\mathrm{B}}$ & $111.77^{\underline{b}}$ & $95.87^{\mathrm{G}}$ & $99.73^{\mathrm{EF}}$ & $101.80^{\mathrm{DE}}$ & $99.13^{\mathrm{e}}$ & $101.82^{\mathrm{f}}$ & $106.55^{\mathrm{cd}}$ & $107.98^{\mathrm{bc}}$ & $105.45^{B}$ \\
\hline & $\mathrm{Sp}_{4}$ & $112.93^{\mathrm{B}}$ & $114.07^{\mathrm{B}}$ & $117.57^{\mathrm{A}}$ & $114.86^{\mathrm{a}}$ & $97.20^{\mathrm{FG}}$ & $103.23^{\mathrm{D}}$ & $103.00^{\mathrm{D}}$ & $101.14^{\mathrm{d}}$ & $105.07^{\mathrm{de}}$ & $108.65^{\mathrm{ab}}$ & $110.28^{\mathrm{a}}$ & $108.00^{A}$ \\
\hline & & $108.90^{\mathrm{B}}$ & $111.94^{\mathrm{AB}}$ & $114.80^{\mathrm{A}}$ & 111.88 & $94.86^{\mathrm{D}}$ & $100.46^{\mathrm{C}}$ & $101.18^{\mathrm{C}}$ & 98.83 & $101.88^{\mathrm{B}}$ & $106.20^{\mathrm{A}}$ & & -- \\
\hline \multicolumn{14}{|c|}{$\begin{array}{l}\text { Mean followed by the same letter are not significantly different at the } 0.05 \text { level by Duncan's multiple range test. } \\
\text { C: Cropping system (Corn-Clover-Wheat \& Corn-fallow-Wheat), } N \text { : } N \text { rates }\left(N_{1}, N_{2} \text { and } N_{3}: \text { application of } 56.25,75.00 \text { and } 93.75 \mathrm{~kg} N / \text { fed, }\right. \\
\text { respectively), Sp: Splitting number ( }\left(\mathrm{Sp}_{2}, \mathrm{Sp}_{3} \text { and } \mathrm{Sp}_{4} \text { : two, three and four equal doses, respectively) }\right.\end{array}$} \\
\hline N & $A B E$ & & & $\mathbf{C} \times \mathbf{N}$ & & & & $\mathbf{N} \times \mathbf{S P}$ & & & abc & & \\
\hline SP & $A B C$ & & & $\mathbf{C} \times \mathbf{S P}$ & & & & $\mathbf{C} \times \mathbf{N} \times \mathbf{S}$ & & & $\mathrm{ABC}$ & & \\
\hline
\end{tabular}

Table 4. Effect of crop sequence, $N$ rates, splitting and their interactions involved on spike length (cm).

\begin{tabular}{|c|c|c|c|c|c|c|c|c|c|c|c|c|c|}
\hline \multirow{2}{*}{ Seasons } & \multirow{2}{*}{$\begin{array}{c}\text { cropping } \\
\text { sequence } \\
\text { N rates }(\mathbf{N}) \\
\text { Splitting }(\mathbf{S p})\end{array}$} & \multicolumn{3}{|c|}{ Corn-Clover-Wheat } & \multirow{2}{*}{ Mean } & \multicolumn{3}{|c|}{ Corn-Fallow-Wheat } & \multirow{2}{*}{ - Mean - } & \multicolumn{3}{|c|}{$\mathbf{N} \times \mathbf{S P}$} & \multirow{2}{*}{ Mean } \\
\hline & & $\mathbf{N}_{1}$ & $\mathbf{N}_{2}$ & $\mathbf{N}_{3}$ & & $\mathbf{N}_{1}$ & $\mathbf{N}_{2}$ & $\mathbf{N}_{3}$ & & $\mathbf{N}_{1}$ & $\mathbf{N}_{2}$ & $\mathbf{N}_{3}$ & \\
\hline \multirow{3}{*}{ 2015-16 } & $\mathrm{Sp}_{2}$ & $10.83^{\mathrm{F}-\mathrm{H}}$ & $11.33^{\mathrm{C}-\mathrm{E}}$ & $11.70^{\mathrm{BC}}$ & $11.29^{\mathrm{c}}$ & $10.00^{1}$ & $10.47^{\mathrm{H}}$ & $10.83^{\mathrm{F}-\mathrm{H}}$ & $10.43^{\mathrm{e}}$ & $10.42^{\mathrm{e}}$ & $10.90^{\mathrm{d}}$ & $11.27^{\mathrm{c}}$ & $10.86^{C}$ \\
\hline & $\mathrm{Sp}_{3}$ & $11.40^{\mathrm{C}-\mathrm{E}}$ & $11.93^{\mathrm{B}}$ & $12.43^{\mathrm{A}}$ & $11.92^{\underline{b}}$ & $10.50^{\mathrm{H}}$ & $10.87^{\mathrm{F}-\mathrm{H}}$ & $11.10^{\mathrm{E}-\mathrm{G}}$ & $10.82^{\mathrm{d}}$ & $10.95^{\mathrm{d}}$ & $11.40^{\mathrm{c}}$ & $11.77^{\mathrm{b}}$ & $11.37^{B}$ \\
\hline & $\mathrm{Sp}_{4}$ & $11.83^{\mathrm{B}}$ & $12.40^{\mathrm{A}}$ & $12.73^{\mathrm{A}}$ & $12.32^{\mathrm{a}}$ & $10.73^{\mathrm{GH}}$ & $11.17^{\mathrm{D}-\mathrm{F}}$ & $11.57^{\mathrm{B}-\mathrm{D}}$ & $11.16^{\mathrm{c}}$ & $11.28^{\mathrm{c}}$ & $11.78^{\mathrm{b}}$ & $12.15^{\mathrm{a}}$ & $11.74^{A}$ \\
\hline \multirow[t]{2}{*}{ Mean } & & $11.36^{\mathrm{B}}$ & $11.89^{\mathrm{A}}$ & $12.29^{\mathrm{A}}$ & 11.84 & $10.41^{\mathrm{D}}$ & $10.83^{\frac{\mathrm{CD}}{\mathrm{D}}}$ & $11.17^{\mathrm{BC}}$ & 10.80 & $10.88^{\epsilon}$ & $11.36^{\mathrm{B}}$ & $11.73^{\mathrm{A}}$ & -- \\
\hline & $\mathrm{Sp}_{2}$ & $11.47^{\mathrm{J}}$ & $11.70^{\mathrm{H}-\mathrm{J}}$ & $12.20^{\mathrm{D}-\mathrm{I}}$ & $11.79^{\mathrm{d}}$ & $10.90^{\mathrm{K}}$ & $11.53^{\mathrm{J}}$ & $11.87^{\mathrm{F}-\mathrm{J}}$ & $11.43^{\mathrm{e}}$ & $11.18^{\mathrm{f}}$ & $11.62^{\mathrm{e}}$ & $12.03^{\mathrm{d}}$ & $11.61^{C}$ \\
\hline \multirow[t]{2}{*}{$2016-17$} & $\mathrm{Sp}_{3}$ & $12.23^{\mathrm{D}-\mathrm{H}}$ & $12.40^{\mathrm{C}-\mathrm{F}}$ & $13.50^{\mathrm{B}}$ & $12.71^{\underline{b}}$ & $11.63^{\mathrm{LJ}}$ & $11.80^{\mathrm{G}-\mathrm{J}}$ & $12.33^{\mathrm{D}-\mathrm{G}}$ & $11.92^{\frac{\mathrm{d}}{}}$ & $11.93^{\mathrm{de}}$ & $12.10^{\mathrm{cd}}$ & $12.92^{\mathrm{b}}$ & $12.32^{B}$ \\
\hline & $\mathrm{Sp}_{4}$ & $12.97^{\mathrm{C}}$ & $13.57^{\mathrm{B}}$ & $14.23^{\mathrm{A}}$ & $13.59^{\mathrm{a}}$ & $11.90^{\mathrm{E}-\mathrm{J}}$ & $12.47^{\mathrm{C}-\mathrm{E}}$ & $12.73^{\mathrm{CD}}$ & $12.37^{\mathrm{c}}$ & $12.43^{\mathrm{c}}$ & $13.02^{\mathrm{b}}$ & $13.48^{\mathrm{a}}$ & $12.98^{A}$ \\
\hline Mean & & $12.22^{\frac{\mathrm{BC}}{\mathrm{C}}}$ & $12.56^{\mathrm{B}}$ & $13.31^{\mathrm{A}}$ & 12.70 & $11.48^{\mathrm{D}}$ & $11.93^{\frac{\mathrm{CD}}{}}$ & $12.31^{\mathrm{BC}}$ & 11.91 & $11.85^{€}$ & $12.24^{\mathrm{B}}$ & $12.81^{\mathrm{A}}$ & -- \\
\hline
\end{tabular}

Table 5. Effect of crop sequence, $\mathbf{N}$ rates, splitting and their interactions involved on number of spike/ $\mathbf{m}^{2}$.

\begin{tabular}{|c|c|c|c|c|c|c|c|c|c|c|c|c|c|}
\hline \multirow[b]{2}{*}{ Seasons } & \multirow{2}{*}{$\begin{array}{c}\text { cropping } \\
\text { sequence } \\
\text { N rates (N) } \\
\text { Splitting } \\
\text { (Sp) }\end{array}$} & \multicolumn{3}{|c|}{ Corn-Clover-Wheat } & \multirow[b]{2}{*}{ Mean } & \multicolumn{3}{|c|}{ Corn-Fallow-Wheat } & \multirow[b]{2}{*}{ Mean } & \multicolumn{3}{|c|}{$\mathbf{N} \times \mathbf{S P}$} & \multirow[b]{2}{*}{ Mean } \\
\hline & & $\mathbf{N}_{1}$ & $\mathbf{N}_{2}$ & $\mathbf{N}_{3}$ & & $\mathbf{N}_{1}$ & $\mathbf{N}_{2}$ & $\mathbf{N}_{3}$ & & $\mathbf{N}_{1}$ & $\mathbf{N}_{2}$ & $\mathbf{N}_{3}$ & \\
\hline \multirow{3}{*}{$2015-16$} & $\mathrm{Sp}_{2}$ & $328.00^{\mathrm{J}}$ & $373.33^{\mathrm{FG}}$ & $420.00^{\mathrm{D}}$ & $373.78^{\mathrm{d}}$ & $305.33^{\mathrm{K}}$ & $334.67^{\mathrm{J}}$ & $368.00^{\mathrm{GH}}$ & $336.00^{\mathrm{e}}$ & $316.67^{\mathrm{h}}$ & $354.00^{\mathrm{g}}$ & $394.00^{\mathrm{d}}$ & $354.89^{C}$ \\
\hline & $\mathrm{Sp}_{3}$ & $354.67^{\mathrm{HI}}$ & $397.33^{\mathrm{E}}$ & $488.00^{\mathrm{B}}$ & $413.33^{\underline{b}}$ & $334.67^{\mathrm{J}}$ & $360.00^{\mathrm{G}-\mathrm{I}}$ & $425.33^{\mathrm{D}}$ & $373.33^{-\mathrm{d}}$ & $344.67^{\mathrm{g}}$ & $378.67^{\mathrm{e}}$ & $456.67^{\mathrm{b}}$ & $393.33^{B}$ \\
\hline & $\mathrm{Sp}_{4}$ & $386.67^{\mathrm{EF}}$ & $434.67^{\mathrm{D}}$ & $530.67^{\mathrm{A}}$ & $450.67^{\mathrm{a}}$ & $344.00^{\mathrm{IJ}}$ & $396.00^{\mathrm{E}}$ & $462.67^{\mathrm{C}}$ & $400.89^{\mathrm{c}}$ & $365.33^{\mathrm{f}}$ & $415.33^{\mathrm{c}}$ & $496.67^{\mathrm{a}}$ & $425.78^{A}$ \\
\hline \multirow[t]{2}{*}{ Mean } & & $356.44^{\mathrm{C}}$ & $401.78^{\underline{B}}$ & $479.56^{\mathrm{A}}$ & 412.59 & $328.00^{\mathrm{D}}$ & $363.56^{\mathrm{C}}$ & $418.67^{\underline{B}}$ & 370.07 & $342.22^{6}$ & $382.67^{\mathrm{B}}$ & $449.11^{\mathrm{A}}$ & - \\
\hline & $\mathrm{Sp}_{2}$ & $345.33^{\mathrm{H}-\mathrm{J}}$ & $384.00^{\mathrm{F}-\mathrm{H}}$ & $474.67^{\mathrm{A-C}}$ & $401.33^{\mathrm{c}}$ & $313.33^{\mathrm{J}}$ & $333.33^{\mathrm{IJ}}$ & $381.33^{\mathrm{F}-\mathrm{I}}$ & $342.67^{\mathrm{d}}$ & $329.33^{\mathrm{e}}$ & $358.67^{\mathrm{e}}$ & $428.00^{\text {cd }}$ & $372.00^{C}$ \\
\hline \multirow[t]{2}{*}{ 2016-17 } & $\mathrm{Sp}_{3}$ & $437.33^{\mathrm{CE}}$ & $462.67^{\mathrm{B}-\mathrm{D}}$ & $496.00^{\mathrm{AB}}$ & $465.33^{\underline{b}}$ & $364.00^{\mathrm{G}-\mathrm{I}}$ & $417.33^{\mathrm{D}-\mathrm{F}}$ & $481.33^{\mathrm{ACC}}$ & $420.89^{\mathrm{c}}$ & $400.67^{d}$ & $440.00^{c}$ & $488.67^{\mathrm{ab}}$ & $443.11^{B}$ \\
\hline & $\mathrm{Sp}_{4}$ & $473.33^{\mathrm{ACC}}$ & $494.67^{\mathrm{AB}}$ & $521.33^{\mathrm{A}}$ & $496.44^{\mathrm{a}}$ & $405.33^{\mathrm{E}-\mathrm{G}}$ & $460.00^{\mathrm{B}-\mathrm{D}}$ & $509.33^{\mathrm{AB}}$ & $458.22^{\underline{b}}$ & $439.33^{\mathrm{c}}$ & $477.33^{\mathrm{b}}$ & $515.33^{\mathrm{a}}$ & $477.33^{A}$ \\
\hline Mean & & $418.67^{\mathrm{BC}}$ & $447.11^{\mathrm{B}}$ & $497.33^{\mathrm{A}}$ & 454.37 & $360.89^{\mathrm{D}}$ & $403.56^{\mathrm{C}}$ & $457.33^{\mathrm{B}}$ & 407.26 & $389.78^{€}$ & $425.33^{\mathrm{B}}$ & $477.33^{A}$ & -- \\
\hline
\end{tabular}


Ayat B. H. Gad et al.

Table 6. Effect of crop sequence, $N$ rates, splitting and their interactions involved on number of grains/spike.

\begin{tabular}{|c|c|c|c|c|c|c|c|c|c|c|c|c|c|}
\hline \multirow{2}{*}{ Seasons } & \multirow{2}{*}{$\begin{array}{c}\text { cropping } \\
\text { sequence }\end{array}$} & \multicolumn{3}{|c|}{ Corn-Clover-Wheat } & \multirow{2}{*}{ Mean } & \multicolumn{3}{|c|}{ Corn-Fallow-Wheat } & \multirow{2}{*}{ - Mean - } & \multicolumn{3}{|c|}{$\mathbf{N} \times \mathbf{S P}$} & \multirow{2}{*}{ Mean } \\
\hline & & $\mathbf{N}_{1}$ & $\mathbf{N}_{2}$ & $\mathbf{N}_{3}$ & & $\mathbf{N}_{1}$ & $\mathbf{N}_{2}$ & $\mathbf{N}_{3}$ & & $\mathbf{N}_{1}$ & $\mathbf{N}_{2}$ & $\mathbf{N}_{3}$ & \\
\hline \multirow{3}{*}{ 2015-16 } & $\mathrm{Sp}_{2}$ & $66.03^{\mathrm{H}-\mathrm{J}}$ & $71.93^{\mathrm{D}-\mathrm{G}}$ & $74.33^{\mathrm{D}-\mathrm{F}}$ & $70.77^{\mathrm{cd}}$ & $62.10^{\top}$ & $65.07^{\mathrm{IJ}}$ & $67.83^{\mathrm{G}-\mathrm{I}}$ & $65.00^{\mathrm{e}}$ & $64.07^{\mathrm{d}}$ & $68.50^{\mathrm{c}}$ & $71.08^{\mathrm{c}}$ & $67.88^{C}$ \\
\hline & $\mathrm{Sp}_{3}$ & $71.30^{\mathrm{D}-\mathrm{H}}$ & $71.73^{\mathrm{D}-\mathrm{G}}$ & $82.77^{\mathrm{AB}}$ & $75.27^{\mathrm{b}}$ & $64.97^{\mathrm{IJ}}$ & $69.27^{\mathrm{E}-\mathrm{I}}$ & $70.47^{\mathrm{E}-\mathrm{I}}$ & $68.23^{\mathrm{d}}$ & $68.13^{\mathrm{c}}$ & $70.50^{\mathrm{c}}$ & $76.62^{\mathrm{b}}$ & $71.75^{B}$ \\
\hline & $\mathrm{Sp}_{4}$ & $74.73^{\mathrm{DE}}$ & $80.37^{\mathrm{BC}}$ & $87.73^{\mathrm{A}}$ & $80.94^{\mathrm{a}}$ & $68.70^{\mathrm{F}-\mathrm{I}}$ & $71.27^{\mathrm{D}-\mathrm{H}}$ & $76.50^{\mathrm{CD}}$ & $72.16^{\mathrm{c}}$ & $71.72^{\mathrm{c}}$ & $75.82^{\mathrm{b}}$ & $82.12^{\mathrm{a}}$ & $76.55^{A}$ \\
\hline Mean & & $70.69^{\frac{\mathrm{BC}}{2}}$ & $74.68^{\underline{B}}$ & $81.61^{\mathrm{A}}$ & 75.66 & $65.26^{\underline{\mathrm{D}}}$ & $68.53^{\mathrm{CD}}$ & $71.60^{\mathrm{BC}}$ & 68.46 & $67.97^{6}$ & $71.61^{\mathrm{B}}$ & $76.61^{\mathrm{A}}$ & -- \\
\hline \multirow{3}{*}{ 2016-17 } & $\mathrm{Sp}_{2}$ & $60.80^{\mathrm{FG}}$ & $62.30^{\mathrm{D}-\mathrm{F}}$ & $63.43^{\mathrm{D}-\mathrm{F}}$ & $62.18^{\mathrm{c}}$ & $53.73^{\mathrm{H}}$ & $53.93^{\mathrm{H}}$ & $60.10^{\mathrm{FG}}$ & $55.92^{\frac{\mathrm{d}}{}}$ & $57.27^{\mathrm{e}}$ & $58.12^{\mathrm{de}}$ & $61.77^{\text {cd }}$ & $59.05^{C}$ \\
\hline & $\mathrm{Sp}_{3}$ & $63.10^{\mathrm{D}-\mathrm{F}}$ & $66.70^{\mathrm{B}-\mathrm{E}}$ & $70.83^{\mathrm{B}}$ & $66.88^{\underline{b}}$ & $56.33^{\mathrm{GH}}$ & $60.37^{\mathrm{FG}}$ & $65.10^{\mathrm{C}-\mathrm{F}}$ & $60.60^{\mathrm{c}}$ & $59.72^{\text {de }}$ & $63.53^{\mathrm{c}}$ & $67.97^{\mathrm{b}}$ & $63.74^{B}$ \\
\hline & $\mathrm{Sp}_{4}$ & $66.87^{\mathrm{B}-\mathrm{E}}$ & $71.93^{\mathrm{B}}$ & $78.17^{\mathrm{A}}$ & $72.32^{\mathrm{a}}$ & $61.50^{\mathrm{E}-\mathrm{G}}$ & $67.43^{\mathrm{B}-\mathrm{D}}$ & $69.60^{\mathrm{BC}}$ & $66.18^{\underline{b}}$ & $64.18^{\mathrm{c}}$ & $69.68^{\mathrm{b}}$ & $73.88^{\mathrm{a}}$ & $69.25^{A}$ \\
\hline Mean & & $63.59^{\mathrm{C}}$ & $66.98^{\underline{B}}$ & $70.81^{\mathrm{A}}$ & 67.13 & $57.19^{\mathrm{E}}$ & $60.58^{\mathrm{D}}$ & $64.93^{\frac{B C}{B}}$ & 60.90 & $60.39^{\epsilon}$ & $63.78^{\mathrm{B}}$ & $67.87^{\mathrm{A}}$ & -- \\
\hline
\end{tabular}

F test for cropping sequence $2015 / 2016 \quad 2016 / 2017$

* and **: significant at $5 \%$ and $1 \%$ level of probability, respectively.

Mean followed by the same letter are not significantly different at the 0.05 level by Duncan's multiple range test.

Table 7. Effect of crop sequence, $\mathbf{N}$ rates, splitting and their interactions involved on weight of grains/spike (g).

\begin{tabular}{|c|c|c|c|c|c|c|c|c|c|c|c|c|c|}
\hline \multirow{2}{*}{ Seasons } & \multirow{2}{*}{$\begin{array}{c}\begin{array}{c}\text { cropping } \\
\text { sequence }\end{array} \\
\mathbf{N} \text { rates }(\mathbf{N}) \\
\text { Splitting }(\mathbf{S p})\end{array}$} & \multicolumn{3}{|c|}{ Corn-Clover-Wheat } & \multirow{2}{*}{ Mean } & \multicolumn{3}{|c|}{ Corn-Fallow-Wheat } & \multirow{2}{*}{ Mean } & \multicolumn{3}{|c|}{$\mathbf{N} \times \mathbf{S P}$} & \multirow{2}{*}{ Mean } \\
\hline & & $\mathbf{N}_{1}$ & $\mathbf{N}_{2}$ & $\mathbf{N}_{3}$ & & $\mathbf{N}_{1}$ & $\mathbf{N}_{2}$ & $\mathbf{N}_{3}$ & & $\mathbf{N}_{1}$ & $\mathbf{N}_{2}$ & $\mathbf{N}_{3}$ & \\
\hline \multirow{3}{*}{$2015-16$} & $\mathrm{Sp}_{2}$ & $2.78^{\mathrm{G}-\mathrm{I}}$ & $2.91^{\mathrm{FG}}$ & $3.11^{\mathrm{EF}}$ & $2.93^{\mathrm{c}}$ & $2.43^{\mathrm{J}}$ & $2.55^{\mathrm{IJ}}$ & $2.65^{\mathrm{H}-\mathrm{J}}$ & $2.54^{\mathrm{e}}$ & $2.60^{\mathrm{f}}$ & $2.73^{\mathrm{ef}}$ & $2.88^{\mathrm{de}}$ & $2.74^{C}$ \\
\hline & $\mathrm{Sp}_{3}$ & $3.22^{\mathrm{DE}}$ & $3.24^{\mathrm{C}-\mathrm{E}}$ & $3.41^{\mathrm{B}-\mathrm{D}}$ & $3.29^{\mathrm{b}}$ & $2.62^{\mathrm{H}-\mathrm{J}}$ & $2.81^{\mathrm{GH}}$ & $2.91^{\mathrm{FG}}$ & $2.78^{\mathrm{d}}$ & $2.92^{\mathrm{d}}$ & $3.03^{\mathrm{cd}}$ & $3.16^{\mathrm{bc}}$ & $3.04^{B}$ \\
\hline & $\mathrm{Sp}_{4}$ & $3.47^{\mathrm{BC}}$ & $3.56^{\mathrm{B}}$ & $3.86^{\mathrm{A}}$ & $3.63^{\mathrm{a}}$ & $2.80^{\mathrm{GH}}$ & $2.92^{\mathrm{FG}}$ & $3.26^{\mathrm{C}-\mathrm{E}}$ & $2.99^{\mathrm{c}}$ & $3.14^{\mathrm{bc}}$ & $3.24^{\mathrm{b}}$ & $3.56^{\mathrm{a}}$ & $3.31^{A}$ \\
\hline \multirow[t]{2}{*}{ Mean } & & $3.16^{\mathrm{B}}$ & $3.24^{\underline{B}}$ & $3.46^{\mathrm{A}}$ & 3.29 & $2.62^{\mathrm{E}}$ & $2.76^{\mathrm{D}}$ & $2.94^{\mathrm{C}}$ & 2.77 & $2.89^{\epsilon}$ & $3.00^{\mathrm{B}}$ & $3.20^{\mathrm{A}}$ & -- \\
\hline & $\mathrm{Sp}_{2}$ & $2.56^{\mathrm{HI}}$ & $2.71^{\mathrm{GH}}$ & $2.97^{\mathrm{C}-\mathrm{F}}$ & $2.75^{\mathrm{d}}$ & $2.48^{\mathrm{I}}$ & $2.60^{\mathrm{HI}}$ & $2.87^{\mathrm{E}-\mathrm{G}}$ & $2.65^{\mathrm{d}}$ & $2.52^{\mathrm{e}}$ & $2.65^{\mathrm{d}}$ & $2.92^{\mathrm{c}}$ & $2.70^{C}$ \\
\hline \multirow[t]{2}{*}{ 2016-17 } & $\mathrm{Sp}_{3}$ & $2.73^{\mathrm{GH}}$ & $2.99^{\mathrm{C}-\mathrm{F}}$ & $3.24^{\mathrm{B}}$ & $2.99^{\mathrm{b}}$ & $2.61^{\mathrm{HI}}$ & $2.82^{\mathrm{FG}}$ & $3.17^{\mathrm{BC}}$ & $2.87^{\mathrm{c}}$ & $2.67^{\mathrm{d}}$ & $2.91^{\mathrm{c}}$ & $3.21^{\mathrm{b}}$ & $2.93^{B}$ \\
\hline & $\mathrm{Sp}_{4}$ & $3.05^{\mathrm{B}-\mathrm{E}}$ & $3.25^{\mathrm{B}}$ & $3.61^{\mathrm{A}}$ & $3.30^{\mathrm{a}}$ & $2.90^{\mathrm{D}-\mathrm{G}}$ & $3.09^{\mathrm{B}-\mathrm{D}}$ & $3.22^{\mathrm{B}}$ & $3.07^{\mathrm{b}}$ & $2.98^{\mathrm{c}}$ & $3.17^{\mathrm{b}}$ & $3.41^{\mathrm{a}}$ & $3.19^{A}$ \\
\hline Mean & & $2.78^{\mathrm{CD}}$ & $2.98^{\mathrm{BC}}$ & $3.27^{\mathrm{A}}$ & 3.01 & $2.66^{\underline{D}}$ & $2.83^{\mathrm{CD}}$ & $3.09^{\mathrm{AB}}$ & 2.86 & $2.72^{\mathrm{G}}$ & $2.91^{\mathrm{B}}$ & $3.18^{\mathrm{A}}$ & -- \\
\hline
\end{tabular}

F test for cropping sequence $2015 / 2016 \quad 2016 / 2017$

*: significant at $5 \%$ level of probability

Mean followed by the same letter are not significantly different at the 0.05 level by Duncan's multiple range test.

Table 8. Effect of crop sequence, $\mathbf{N}$ rates, splitting and their interactions involved on seed index (g).

\begin{tabular}{|c|c|c|c|c|c|c|c|c|c|c|c|c|c|}
\hline \multirow{2}{*}{ Seasons } & \multirow{2}{*}{$\begin{array}{c}\begin{array}{c}\text { cropping } \\
\text { sequence }\end{array} \\
\text { N rates }(\mathbf{N}) \\
\text { Splitting (Sp) }\end{array}$} & \multicolumn{3}{|c|}{ Corn-Clover-Wheat } & \multirow{2}{*}{ Mean } & \multicolumn{3}{|c|}{ Corn-Fallow-Wheat } & \multirow{2}{*}{ Mean } & \multicolumn{3}{|c|}{$\mathbf{N} \times \mathbf{S P}$} & \multirow{2}{*}{ Mean } \\
\hline & & $\mathbf{N}_{1}$ & $\mathbf{N}_{2}$ & $\mathbf{N}_{3}$ & & $\mathbf{N}_{1}$ & $\mathbf{N}_{2}$ & $\mathbf{N}_{3}$ & & $\mathbf{N}_{1}$ & $\mathbf{N}_{2}$ & $\mathbf{N}_{3}$ & \\
\hline \multirow{3}{*}{$2015-16$} & $\mathrm{Sp}_{2}$ & $43.75^{\mathrm{E}-\mathrm{G}}$ & $47.85^{\mathrm{CD}}$ & $48.10^{\mathrm{CD}}$ & $46.57^{\mathrm{c}}$ & $40.53^{\mathrm{J}}$ & $41.72^{\mathrm{H}-\mathrm{J}}$ & $42.85^{\mathrm{F}-\mathrm{H}}$ & $41.70^{\mathrm{e}}$ & $42.14^{\mathrm{f}}$ & $44.78^{\mathrm{de}}$ & $45.48^{\mathrm{d}}$ & $44.13^{C}$ \\
\hline & $\mathrm{Sp}_{3}$ & $46.95^{\mathrm{D}}$ & $49.10^{\mathrm{BC}}$ & $50.02^{\mathrm{B}}$ & $48.69^{\mathrm{b}}$ & $41.15^{\mathrm{IJ}}$ & $42.62^{\mathrm{G}-\mathrm{I}}$ & $43.42^{\mathrm{E}-\mathrm{G}}$ & $42.39^{\mathrm{e}}$ & $44.05^{\mathrm{e}}$ & $45.86^{\mathrm{cd}}$ & $46.72^{\mathrm{bc}}$ & $45.54^{B}$ \\
\hline & $\mathrm{Sp}_{4}$ & $48.18^{\mathrm{CD}}$ & $49.75^{\mathrm{B}}$ & $52.87^{\mathrm{A}}$ & $50.27^{\mathrm{a}}$ & $42.37^{\mathrm{G}-\mathrm{I}}$ & $44.38^{\mathrm{EF}}$ & $44.70^{\mathrm{E}}$ & $43.82^{-\mathrm{d}}$ & $45.28^{d}$ & $47.07^{\mathrm{b}}$ & $48.78^{\mathrm{a}}$ & $47.04^{A}$ \\
\hline \multirow[t]{2}{*}{ Mean } & & $46.29^{\mathrm{C}}$ & $48.90^{\mathrm{B}}$ & $50.33^{\mathrm{A}}$ & 48.51 & $41.35^{\mathrm{E}}$ & $42.91^{\mathrm{D}}$ & $43.66^{-\mathrm{D}}$ & 42.64 & $43.82^{\epsilon}$ & $45.90^{\mathrm{B}}$ & $46.99^{A}$ & -- \\
\hline & $\mathrm{Sp}_{2}$ & $43.47^{\mathrm{J}-\mathrm{L}}$ & $45.58^{\mathrm{E}-\mathrm{I}}$ & $46.75^{\mathrm{B}-\mathrm{E}}$ & $45.27^{\mathrm{cd}}$ & $42.37^{\mathrm{L}}$ & $44.08^{1-K}$ & $44.77^{\mathrm{G}-\mathrm{J}}$ & $43.74^{\mathrm{e}}$ & $42.92^{\mathrm{e}}$ & $44.83^{\text {cd }}$ & $45.76^{\mathrm{c}}$ & $44.50^{C}$ \\
\hline \multirow[t]{2}{*}{ 2016-17 } & $\mathrm{Sp}_{3}$ & $45.73^{\mathrm{D}-\mathrm{H}}$ & $46.70^{\mathrm{B}-\mathrm{E}}$ & $47.88^{\mathrm{BC}}$ & $46.77^{\underline{b}}$ & $43.05^{\mathrm{KL}}$ & $44.93^{\mathrm{F-J}}$ & $46.50^{\mathrm{C}-\mathrm{F}}$ & $44.83^{\mathrm{d}}$ & $44.39^{d}$ & $45.82^{\mathrm{c}}$ & $47.19^{b}$ & $45.80^{B}$ \\
\hline & $\mathrm{Sp}_{4}$ & $47.10^{\mathrm{B}-\mathrm{E}}$ & $48.25^{\mathrm{B}}$ & $50.05^{\mathrm{A}}$ & $48.47^{\mathrm{a}}$ & $44.55^{\mathrm{H}-\mathrm{K}}$ & $46.32^{\mathrm{C}-\mathrm{G}}$ & $47.30^{\mathrm{B}-\mathrm{D}}$ & $46.06^{\mathrm{bc}}$ & $45.83^{\mathrm{c}}$ & $47.28^{b}$ & $48.68^{\mathrm{a}}$ & $47.26^{A}$ \\
\hline Mean & & $45.43^{\frac{\mathrm{BC}}{}}$ & $46.84^{\mathrm{AB}}$ & $48.23^{\mathrm{A}}$ & 46.84 & $43.32^{\underline{D}}$ & $45.11^{\mathrm{C}}$ & $46.19^{\mathrm{BC}}$ & 44.87 & $44.38^{€}$ & $45.98^{\mathrm{B}}$ & $47.21^{\mathrm{A}}$ & -- \\
\hline
\end{tabular}

F test for cropping sequence 2015/2016 2016/2017

$* *$ : significant at $1 \%$ level of probability

Mean followed by the same letter are not significantly different at the 0.05 level by Duncan's multiple range test.

The highest mean values of these traits were obtained from the sequence of corn-clover-wheat with high $\mathrm{N}$ rate $(93.75 \mathrm{~kg} \mathrm{~N} / \mathrm{fed})$ when it applied at four equal doses in both seasons.

Grain and straw yield traits:

The results obtained in Tables 9 and 10 emphasize clearly that the effect of cropping sequence on grain and straw yield was significant in both seasons. The data showed that the sequence of corn-clover-wheat produced highest grain and straw yield when compared with the sequence of corn-fallow-wheat. The highest mean values of mentioned traits (23.77 ard /fed and 5.13 ton/fed of grain and straw yield in the first season, respectively being $24.47 \mathrm{ard} /$ fed and $5.97 \mathrm{ton} / \mathrm{fed}$ in the second season in the same order) were produced from the sequence of corn-clover-wheat. The present trend is to be expected from the yield attributes had the same line. These results are in accordance with those obtained by Said (2007), Dogan and Bilgili (2010), Hefny (2012) and Zaheer et al. (2015).

Regarding nitrogen fertilizer rates the results in the same Tables show that grain and straw yield were significantly affected by nitrogen fertilizer rates in both seasons. Application of $93.75 \mathrm{~kg} \mathrm{~N} / \mathrm{fed}$ achieved the highest value of these traits (24.55 ard /fed and 4.95 ton/fed of grain and straw yield in the first season, respectively being $24.18 \mathrm{ard} / \mathrm{fed}$ and $5.60 \mathrm{ton} / \mathrm{fed}$ in the second season in the same order). The present trend is to be expected from the yield attributes had the same line. The increments in yield and its attributes of wheat with increasing $\mathrm{N}$ rates up to adequate $\mathrm{N}$ need might be attributed to the effective role of $\mathrm{N}$ as an essential constituent of chlorophyll on dry matter accumulation. $\mathrm{N}$ fertilizer influences the production 
of carbohydrates by affecting the mean leaf area available to intercept solar radiation and absorb $\mathrm{CO}_{2}$, promoting the efficiency of photosynthesis process. Also, the significant enhancement of yield components in increasing order with different nitrogen rates can be credited to higher dose of nitrogen, which greatly helps the plant to expose its potential to grow potentially. These results are in general agree with those reported by Abedi et al. (2011), El-Agrodi et al. (2011), Haile et al. (2012), Mandic et al. (2015), Zaheer et al. (2015), Mansour et al. (2016), El-Temsah (2017) and Haque et al. (2017).

From the data presented in the same Tables indicate that the effect of split nitrogen fertilizer on straw and grain yield was significant in both seasons. Grain and straw yield from four equal split doses of $\mathrm{N}$ applications were generally better than of two or three equal split doses. Additionally, these traits of three applications were superior to two applications of $\mathrm{N}$. The highest mean values of these characters (23.58 ard /fed and 4.90 ton/fed of grain and straw yield in the first season, respectively being 24.74 $\operatorname{ard} /$ fed and 5.47 ton/fed in the second season in the same order) was recorded when $\mathrm{N}$ fertilizer was applied in four equal split doses before the second, third, fourth and fifth irrigations. The present trend is to be expected from the yield attributes had the same line. These results are confirmed by Abedi et al. (2011), El-Agrodi et al. (2011), Haile et al. (2012), Ercoli et al. (2013), Usman and UrRehman (2014) and Singh et al. (2016).

Table 9. Effect of cropping sequence, $N$ rates, splitting and their interactions involved on grain yield ardab/fed.

\begin{tabular}{|c|c|c|c|c|c|c|c|c|c|c|c|c|c|}
\hline \multirow{2}{*}{ Seasons } & \multirow{2}{*}{$\begin{array}{c}\text { cropping } \\
\text { sequence } \\
\text { N rates }(\mathbf{N}) \\
\text { Splitting }(\mathbf{S p})\end{array}$} & \multicolumn{3}{|c|}{ Corn-Clover-Wheat } & \multirow{2}{*}{ Mean } & \multicolumn{3}{|c|}{ Corn-Fallow-Wheat } & \multirow{2}{*}{ Mean } & \multicolumn{3}{|c|}{$\mathbf{N} \times \mathbf{S P}$} & \multirow{2}{*}{ Mean } \\
\hline & & $\mathbf{N}_{1}$ & $\mathbf{N}_{2}$ & $\mathbf{N}_{3}$ & & $\mathbf{N}_{1}$ & $\mathbf{N}_{2}$ & $\mathbf{N}_{3}$ & & $\mathbf{N}_{1}$ & $\mathbf{N}_{2}$ & $\mathbf{N}_{3}$ & \\
\hline \multirow{3}{*}{ 2015-16 } & $\mathrm{Sp}_{2}$ & $19.69^{\mathrm{I}-\mathrm{K}}$ & $21.78^{\mathrm{E}-\mathrm{G}}$ & $24.62^{\mathrm{C}}$ & $22.03^{\mathrm{c}}$ & $17.11^{\mathrm{L}}$ & $18.89^{\mathrm{JK}}$ & $20.93^{\mathrm{G}-\mathrm{I}}$ & $18.98^{\mathrm{e}}$ & $18.40^{\mathrm{g}}$ & $20.33^{\mathrm{f}}$ & $22.78^{\mathrm{cd}}$ & $20.50^{\mathrm{C}}$ \\
\hline & $\mathrm{Sp}_{3}$ & $22.04^{\mathrm{E}-\mathrm{G}}$ & $23.82^{\mathrm{CD}}$ & $26.40^{\mathrm{AB}}$ & $24.09^{\mathrm{b}}$ & $18.67^{\mathrm{K}}$ & $20.22^{\mathrm{H}-\mathrm{J}}$ & $23.02^{\mathrm{DE}}$ & $20.64^{\frac{d}{d}}$ & $20.36^{\mathrm{f}}$ & $22.02^{\text {de }}$ & $24.71^{\mathrm{b}}$ & $22.36^{B}$ \\
\hline & $\mathrm{Sp}_{4}$ & $22.84^{\mathrm{D}-\mathrm{F}}$ & $25.29^{\mathrm{BC}}$ & $27.47^{\mathrm{A}}$ & $25.20^{\mathrm{a}}$ & $19.60^{\mathrm{I}-\mathrm{K}}$ & $21.42^{\mathrm{F}-\mathrm{H}}$ & $24.84^{\mathrm{C}}$ & $21.96^{\mathrm{c}}$ & $21.22^{\mathrm{ef}}$ & $23.36^{\mathrm{c}}$ & $26.16^{\mathrm{a}}$ & $23.58^{A}$ \\
\hline \multirow[t]{2}{*}{ Mean } & & $21.53^{\frac{\mathrm{BC}}{}}$ & $23.63^{\underline{B}}$ & $26.16^{\mathrm{A}}$ & 23.77 & $18.46^{\mathrm{D}}$ & $20.18^{\underline{C D}}$ & $22.93^{\underline{B}}$ & 20.52 & $19.99^{\mathrm{C}}$ & $21.90^{\mathrm{B}}$ & $24.55^{\mathrm{A}}$ & -- \\
\hline & $\mathrm{Sp}_{2}$ & $20.67^{\mathrm{F}-\mathrm{H}}$ & $21.38^{\mathrm{E}-\mathrm{H}}$ & $25.02^{\mathrm{B}-\mathrm{D}}$ & $22.36^{\mathrm{c}}$ & $16.18^{\mathrm{I}}$ & $18.98^{\mathrm{H}}$ & $20.40^{\mathrm{F}-\mathrm{H}}$ & $18.52^{\mathrm{e}}$ & $18.42^{\mathrm{f}}$ & $20.18^{\mathrm{e}}$ & $22.71^{\mathrm{cd}}$ & $20.44^{C}$ \\
\hline \multirow[t]{2}{*}{ 2016-17 } & $\mathrm{Sp}_{3}$ & $23.07^{\mathrm{D}-\mathrm{F}}$ & $23.07^{\mathrm{D}-\mathrm{F}}$ & $25.73^{\mathrm{A}-\mathrm{C}}$ & $23.96^{\mathrm{b}}$ & $19.91^{\mathrm{GH}}$ & $20.67^{\mathrm{F}-\mathrm{H}}$ & $22.00^{\mathrm{E}-\mathrm{G}}$ & $20.86^{\mathrm{d}}$ & $21.49^{\text {de }}$ & $21.87^{\text {de }}$ & $23.87^{\mathrm{bc}}$ & $22.41^{B}$ \\
\hline & $\mathrm{Sp}_{4}$ & $26.18^{\mathrm{A}-\mathrm{C}}$ & $26.89^{\mathrm{AB}}$ & $28.22^{\mathrm{A}}$ & $27.10^{\mathrm{a}}$ & $21.38^{\mathrm{E}-\mathrm{H}}$ & $22.09^{\mathrm{E}-\mathrm{G}}$ & $23.69^{\mathrm{C}-\mathrm{E}}$ & $22.39^{\mathrm{c}}$ & $23.78^{\mathrm{bc}}$ & $24.49^{\mathrm{ab}}$ & $25.96^{\mathrm{a}}$ & $24.74^{A}$ \\
\hline Mean & & $23.30^{\frac{\mathrm{BC}}{\mathrm{C}}}$ & $23.78^{\mathrm{AB}}$ & $26.33^{\mathrm{A}}$ & 24.47 & $19.16^{\underline{\mathrm{D}}}$ & $20.58^{\frac{\mathrm{CD}}{}}$ & $22.03^{\frac{B-D}{1}}$ & 20.59 & $21.23^{\mathrm{B}}$ & $22.18^{\mathrm{B}}$ & $24.18^{\mathrm{A}}$ & -- \\
\hline
\end{tabular}

F test for cropping sequence $2015 / 2016 \quad 2016 / 2017$

$*$ and $* *$ : significant at $5 \%$ and $1 \%$ level of probability, respectively.

Mean followed by the same letter are not significantly different at the 0.05 level by Duncan's multiple range test.

Table 10. Effect of cropping sequence, $\mathbf{N}$ rates, splitting and their interactions involved on straw yield ton/fed.

\begin{tabular}{|c|c|c|c|c|c|c|c|c|c|c|c|c|c|}
\hline \multirow{2}{*}{ Seasons } & \multirow{2}{*}{$\begin{array}{c}\begin{array}{c}\text { cropping } \\
\text { sequence }\end{array} \\
\text { N rates }(\mathbf{N}) \\
\text { Splitting }(\mathrm{Sp})\end{array}$} & \multicolumn{3}{|c|}{ Corn-Clover-Wheat } & \multirow{2}{*}{ Mean } & \multicolumn{3}{|c|}{ Corn-Fallow-Wheat } & \multirow{2}{*}{ Mean } & \multicolumn{3}{|c|}{$\mathbf{N} \times \mathbf{S P}$} & \multirow{2}{*}{ Mean } \\
\hline & & $\mathbf{N}_{1}$ & $\mathbf{N}_{2}$ & $\mathbf{N}_{3}$ & & $\mathbf{N}_{1}$ & $\mathbf{N}_{2}$ & $\mathbf{N}_{3}$ & & $\mathbf{N}_{1}$ & $\mathbf{N}_{2}$ & $\mathbf{N}_{3}$ & \\
\hline \multirow{3}{*}{ 2015-16 } & $\mathrm{Sp}_{2}$ & $4.96^{\mathrm{B}-\mathrm{D}}$ & $4.87^{\mathrm{CD}}$ & $5.37^{\mathrm{AB}}$ & $5.07^{\mathrm{a}}$ & $3.81^{\mathrm{F}}$ & $3.83^{\mathrm{EF}}$ & $4.22^{\mathrm{EF}}$ & $3.95^{\mathrm{c}}$ & $4.39^{\mathrm{cd}}$ & $4.35^{\mathrm{d}}$ & $4.80^{\mathrm{b}}$ & $4.51^{B}$ \\
\hline & $\mathrm{Sp}_{3}$ & $4.91^{\mathrm{CD}}$ & $4.89^{\mathrm{CD}}$ & $5.30^{\mathrm{A}-\mathrm{C}}$ & $5.04^{\mathrm{a}}$ & $3.90^{\mathrm{EF}}$ & $4.01^{\mathrm{EF}}$ & $4.08^{\mathrm{EF}}$ & $4.00^{\mathrm{c}}$ & $4.41^{\mathrm{cd}}$ & $4.45^{\mathrm{cd}}$ & $4.69^{b c}$ & $4.52^{B}$ \\
\hline & $\mathrm{Sp}_{4}$ & $4.79^{\mathrm{D}}$ & $5.41^{\mathrm{AB}}$ & $5.63^{\mathrm{A}}$ & $5.28^{\mathrm{a}}$ & $4.28^{\mathrm{E}}$ & $4.19^{\mathrm{EF}}$ & $5.12^{\mathrm{B}-\mathrm{D}}$ & $4.53^{\mathrm{b}}$ & $4.54^{\mathrm{b}-\mathrm{d}}$ & $4.80^{\mathrm{b}}$ & $5.38^{\mathrm{a}}$ & $4.90^{A}$ \\
\hline \multirow[t]{2}{*}{ Mean } & & $4.89^{\mathrm{BC}}$ & $5.06^{\mathrm{AB}}$ & $5.44^{\mathrm{A}}$ & 5.13 & $4.00^{\mathrm{D}}$ & $4.01^{\mathrm{D}}$ & $4.47^{\mathrm{C}}$ & 4.16 & $4.44^{\mathrm{B}}$ & $4.53^{\mathrm{B}}$ & $4.95^{\mathrm{A}}$ & -- \\
\hline & $\mathrm{Sp}_{2}$ & $5.37^{\mathrm{C}-\mathrm{E}}$ & $5.83^{\mathrm{B}-\mathrm{D}}$ & $6.24^{\mathrm{AB}}$ & $5.81^{\mathrm{a}}$ & $4.28^{\mathrm{G}}$ & $4.30^{\mathrm{G}}$ & $4.53^{\mathrm{E}-\mathrm{G}}$ & $4.37^{\underline{b}}$ & $4.82^{\mathrm{c}}$ & $5.06^{\mathrm{bc}}$ & $5.39^{\mathrm{a}-\mathrm{c}}$ & $5.09^{B}$ \\
\hline \multirow[t]{2}{*}{ 2016-17 } & $\mathrm{Sp}_{3}$ & $5.76^{\mathrm{B}-\mathrm{D}}$ & $6.05^{\mathrm{A}-\mathrm{C}}$ & $6.01^{\mathrm{A}-\mathrm{C}}$ & $5.94^{\mathrm{a}}$ & $4.49^{\mathrm{FG}}$ & $4.55^{\mathrm{E}-\mathrm{G}}$ & $5.15^{\mathrm{D}-\mathrm{F}}$ & $4.73^{\underline{b}}$ & $5.13^{\mathrm{bc}}$ & $5.30^{\mathrm{a}-\mathrm{c}}$ & $5.58^{\mathrm{ab}}$ & $5.34^{A B}$ \\
\hline & $\mathrm{Sp}_{4}$ & $6.22^{\mathrm{AB}}$ & $5.63^{\mathrm{B}-\mathrm{D}}$ & $6.67^{\mathrm{A}}$ & $6.17^{\mathrm{a}}$ & $4.63^{\mathrm{E}-\mathrm{G}}$ & $4.69^{\mathrm{E}-\mathrm{G}}$ & $5.00^{\mathrm{D}-\mathrm{G}}$ & $4.77^{\underline{b}}$ & $5.42^{\mathrm{ab}}$ & $5.16^{\mathrm{bc}}$ & $5.83^{\mathrm{a}}$ & $5.47^{A}$ \\
\hline Mean & & $5.78^{\mathrm{A}}$ & $5.83^{\mathrm{A}}$ & $6.30^{\mathrm{A}}$ & 5.97 & $4.47^{\underline{B}}$ & $4.51^{\mathrm{B}}$ & $4.90^{\mathrm{B}}$ & 4.63 & $5.12^{\mathrm{B}}$ & $5.17^{\mathrm{B}}$ & $5.60^{\mathrm{A}}$ & -- \\
\hline
\end{tabular}

F test for cropping sequence $2015 / 2016 \quad 2016 / 2017$

$*$ and $* *$ : significant at $5 \%$ and $1 \%$ level of probability, respectively.

Mean followed by the same letter are not significantly different at the 0.05 level by Duncan's multiple range test.

In case of interaction effects in this respect, the data presented show that the first order interaction of cropping sequence $\times \mathrm{N}$ rates proved to be significant on grain and straw yield in both seasons. The highest mean values of these traits were obtained from the sequence of corn-clover wheat when received $93.75 \mathrm{~kg} \mathrm{~N} /$ fed in both seasons. Here too, the first order interaction of cropping sequence $\times$ splitting number turned to be significant in this respect in both seasons. The highest mean values of these traits were obtained from the sequence of corn-clover-wheat with four equal split doses of $\mathrm{N}$ application in both seasons. Also, the first order interaction of $\mathrm{N}$ rates $\times$ splitting number proved to be significant in this respect in both seasons. The highest mean values of these traits were obtained from wheat plants fertilized by $93.75 \mathrm{~kg} \mathrm{~N} /$ fed applied at four equal split doses in both seasons. In addition, the second order interaction was significant in this respect in both seasons. The highest mean values of these traits were obtained from the sequence of corn-clover-wheat with highest $\mathrm{N}$ rate $(93.75 \mathrm{~kg} \mathrm{~N} / \mathrm{fed})$ when it applied at four equal doses in both seasons.

\section{Nitrogen Use Efficiency (NUE):}

Results recorded in Table 11 show that nitrogen use efficiency tended to be reacted significantly to cropping sequence in the second season only. However, the data in the first season was not significant. Planting wheat after clover improved this trait as compared with planting wheat after fallow. The highest mean values of NUE (48.84 and $50.61 \mathrm{~kg}$ grains $/ \mathrm{kg} \mathrm{N}$ applied in the first and second seasons, respectively) were produced from the sequence of corn-clover-wheat. The lowest mean value of NUE (42.09 
and $42.49 \mathrm{~kg}$ grains $/ \mathrm{kg} \mathrm{N}$ applied in the first and second seasons, respectively) was obtained from the sequence of corn-fallow-wheat. These results are in agreement with those mentioned by Rahimizadeh et al. (2010).

The application of $\mathrm{N}$ fertilizer rates had a significant influence on NUE in the two growing seasons. Increasing nitrogen rates decreased NUE values although higher $\mathrm{N}$ rates generally led to higher yield. Application of $56.25 \mathrm{~kg} \mathrm{~N} / \mathrm{fed}$ were more effective compared with the other $\mathrm{N}$ rates in both seasons. Maximum mean values of nitrogen use efficiency (53.31 and $56.61 \mathrm{~kg}$ grains $/ \mathrm{kg} \mathrm{N}$ in the first and second seasons, respectively) were noted when $56.25 \mathrm{~kg} \mathrm{~N} /$ fed was used. The minimum mean values of nitrogen use efficiency (39.28 and $38.68 \mathrm{~kg}$ grains $/ \mathrm{kg} \mathrm{N}$ in the first and second seasons, respectively) were recorded when $93.75 \mathrm{~kg} \mathrm{~N} / \mathrm{fed}$ was applied in both seasons. NUE was reduced in highest $\mathrm{N}$ level. This can be attributed to $\mathrm{N}$ loss in ecosystem. These results are in line with those obtained by Haile et al. (2012), Mandic et al. (2015) and Haque et al. (2017).

Illustrated data in the same Table reveal that split nitrogen fertilizer had a significant influence on nitrogen use efficiency in the two growing seasons. Nitrogen use efficiency was increased by increasing splitting numbers. Splitting $\mathrm{N}$ into four equal doses resulted in the highest mean values of NUE (48.38 and $51.30 \mathrm{~kg}$ grains $/ \mathrm{kg} \mathrm{N}$ applied in first and second seasons, respectively). The lowest mean values of NUE (42.06 and $41.94 \mathrm{~kg}$ grains $/ \mathrm{kg}$ $\mathrm{N}$ applied in first and second seasons, respectively) were observed when application the fertilizer in two equal split doses before the second and third irrigations. It is clear from these data that when a large percentage of nitrogen is used before the second and third irrigations, the loss of nitrate due to leaching in early irrigations increases and nitrogen use efficiency decreases. Therefore, several application of nitrogen fertilizer at different growth stages is highly important for increasing nitrogen use efficiency. It seems that the efficient utilization of nitrogen by plants reflected to higher grain yield and highest mean values of NUE. These results are in the same context of those obtained by Haile et al. (2012) and Ercoli et al. (2013).

Concerning the interactions involved in this respect, the data presented in the same Table show that the first order interaction of cropping sequence $\times \mathrm{N}$ rates proved to be significant in the two growing seasons. The highest mean values of NUE (57.40 and $62.14 \mathrm{~kg}$ grains $/ \mathrm{kg} \mathrm{N}$ applied in first and second seasons, respectively) was obtained from the sequence of corn-clover-wheat when received $56.25 \mathrm{~kg} \mathrm{~N} / \mathrm{fed}$. Here too, the first order interaction of cropping sequence $\times$ splitting number turned to be significant in this respect in both seasons. The highest mean value of NUE (51.81 and $56.25 \mathrm{~kg}$ grains $/ \mathrm{kg} \mathrm{N}$ applied in first and second seasons, respectively) was obtained from the sequence of corn-clover-wheat with four equal doses of $\mathrm{N}$ application. Also, the first order interaction of $\mathrm{N}$ rates $\times$ splitting number proved to be significant in this respect through the two growing seasons. The highest mean values of NUE $(56.59$ and $63.41 \mathrm{~kg}$ grains $/ \mathrm{kg} \mathrm{N}$ applied in first and second seasons, respectively) was obtained from wheat plants fertilized by $56.25 \mathrm{~kg} \mathrm{~N} /$ fed applied at four equal doses. In addition, the second order interaction was significant in this respect in both seasons. The highest mean values of NUE (60.92 and $69.81 \mathrm{~kg}$ grains $/ \mathrm{kg} \mathrm{N}$ applied in the first and second seasons, respectively) was obtained from the sequence of corn-clover-wheat with lowest $\mathrm{N}$ rate $(56.25 \mathrm{~kg} \mathrm{~N} / \mathrm{fed})$ when it applied at four equal doses.

Table 11. Effect of crop sequence, $\mathrm{N}$ rates, splitting and their interactions involved on NUE (kg grains/kg N).

\begin{tabular}{|c|c|c|c|c|c|c|c|c|c|c|c|c|c|}
\hline \multirow{2}{*}{ Seasons } & \multirow{2}{*}{$\begin{array}{c}\begin{array}{c}\text { cropping } \\
\text { sequence }\end{array} \\
\text { N rates }(\mathbf{N}) \\
\text { Splitting }(\mathbf{S p})\end{array}$} & \multicolumn{3}{|c|}{ Corn-Clover-Wheat } & \multirow{2}{*}{ Mean } & \multicolumn{3}{|c|}{ Corn-Fallow-Wheat } & \multirow{2}{*}{ Mean } & \multicolumn{3}{|c|}{$\mathbf{N} \times \mathbf{S P}$} & \multirow{2}{*}{ Mean } \\
\hline & & $\mathbf{N}_{1}$ & $\mathbf{N}_{2}$ & $\mathbf{N}_{3}$ & & $\mathbf{N}_{1}$ & $\mathbf{N}_{2}$ & $\mathbf{N}_{3}$ & & $\mathbf{N}_{1}$ & $\mathbf{N}_{2}$ & $\mathbf{N}_{3}$ & \\
\hline \multirow{3}{*}{$2015-16$} & $\mathrm{Sp}_{2}$ & $52.50^{\mathrm{B}}$ & $43.56^{\mathrm{EF}}$ & $39.40^{\mathrm{H}-\mathrm{J}}$ & $45.15^{\mathrm{c}}$ & $45.63^{\mathrm{DE}}$ & $37.78^{\mathrm{IJ}}$ & $33.49^{\mathrm{K}}$ & $38.97^{\mathrm{e}}$ & $49.07^{\mathrm{c}}$ & $40.67^{\mathrm{fg}}$ & $36.44^{\mathrm{h}}$ & $42.06^{C}$ \\
\hline & $\mathrm{Sp}_{3}$ & $58.79^{\mathrm{A}}$ & $47.64^{\mathrm{CD}}$ & $42.24^{\mathrm{F}-\mathrm{H}}$ & $49.56^{\mathrm{b}}$ & $49.78^{\mathrm{BC}}$ & $40.44^{\mathrm{G}-\mathrm{I}}$ & $36.84^{\mathrm{J}}$ & $42.35^{\mathrm{d}}$ & $54.28^{\mathrm{b}}$ & $44.04^{\mathrm{e}}$ & $39.54^{\mathrm{g}}$ & $45.95^{B}$ \\
\hline & $\mathrm{Sp}_{4}$ & $60.92^{\mathrm{A}}$ & $50.58^{\mathrm{BC}}$ & $43.95^{\mathrm{EF}}$ & $51.81^{\mathrm{a}}$ & $52.27^{\mathrm{B}}$ & $42.84^{\mathrm{E}-\mathrm{G}}$ & $39.75^{\mathrm{H}-\mathrm{J}}$ & $44.95^{\mathrm{c}}$ & $56.59^{\mathrm{a}}$ & $46.71^{\mathrm{d}}$ & $41.85^{\mathrm{f}}$ & $48.38^{A}$ \\
\hline \multirow[t]{2}{*}{ Mean } & & $57.40^{\mathrm{A}}$ & $47.26^{\underline{B}}$ & $41.86^{\mathrm{C}}$ & 48.84 & $49.22^{\underline{B}}$ & $40.36^{\mathrm{CD}}$ & $36.69^{\mathrm{D}}$ & 42.09 & $53.31^{\mathrm{A}}$ & $43.81^{\mathrm{B}}$ & $39.28^{\epsilon}$ & -- \\
\hline & $\mathrm{Sp}_{2}$ & $55.11^{\mathrm{C}}$ & $42.76^{\mathrm{DE}}$ & $40.04^{\mathrm{D}-\mathrm{F}}$ & $45.97^{\mathrm{c}}$ & $43.14^{\mathrm{DE}}$ & $37.96^{\mathrm{E}-\mathrm{G}}$ & $32.64^{\mathrm{G}}$ & $37.91^{\underline{d}}$ & $49.13^{\mathrm{c}}$ & $40.36^{\mathrm{de}}$ & $36.34^{f}$ & $41.94^{C}$ \\
\hline \multirow[t]{2}{*}{ 2016-17 } & $\mathrm{Sp}_{3}$ & $61.51^{\mathrm{B}}$ & $46.13^{\mathrm{D}}$ & $41.17^{\mathrm{DE}}$ & $49.61^{\underline{b}}$ & $53.10^{\mathrm{C}}$ & $41.33^{\mathrm{DE}}$ & $35.20^{\mathrm{FG}}$ & $43.21^{\mathrm{c}}$ & $57.30^{\mathrm{b}}$ & $43.73^{\mathrm{d}}$ & $38.19^{\mathrm{ef}}$ & $46.41^{B}$ \\
\hline & $\mathrm{Sp}_{4}$ & $69.81^{\mathrm{A}}$ & $53.78^{\mathrm{C}}$ & $45.16^{\mathrm{D}}$ & $56.25^{\mathrm{a}}$ & $57.01^{\mathrm{BC}}$ & $44.18^{\mathrm{D}}$ & $37.90^{\mathrm{E}-\mathrm{G}}$ & $46.36^{\mathrm{c}}$ & $63.41^{\mathrm{a}}$ & $48.98^{\mathrm{c}}$ & $41.53^{\mathrm{de}}$ & $51.30^{A}$ \\
\hline Mean & & $62.14^{\mathrm{A}}$ & $47.56^{\mathrm{BC}}$ & $42.12^{\frac{\mathrm{CD}}{}}$ & 50.61 & $51.08^{\underline{B}}$ & $41.16^{\mathrm{DE}}$ & $35.25^{\mathrm{E}}$ & 42.49 & $56.61^{\mathrm{A}}$ & $44.36^{\mathrm{B}}$ & $38.68^{\epsilon}$ & -- \\
\hline
\end{tabular}

F test for cropping sequence 2015/2016 2016/2017

NS and *: not significant and significant at $5 \%$ level of probability, respectively.

Mean followed by the same letter are not significantly different at the 0.05 level by Duncan's multiple range test.

\section{CONCLUSION}

From the obtained results the highest wheat yield obtained by planting wheat after clover and fertilized it by $93.75 \mathrm{~kg} \mathrm{~N} / \mathrm{fed}$ added at four equal doses under the same condition.

\section{REFERENCES}

Ali, E. A. (2011): Impact of nitrogen application time on grain and protein yields as well as nitrogen use efficiency of some two-row barley cultivars in sandy soil. American-Eurasian J. Agric. and Environ. Sci., 10 (3): 425-433.
Abedi, T.; A. Alemzadeh and S. A. Kazemeini (2011): Wheat yield and grain protein response to nitrogen amount and timing. Australian J. Crop Sci., 5 (3):330-336.

CoStat Version 6.303 Copyright (1998-2004) CoHort Software798 Lighthouse Ave. PMB 320, Monterey, CA, 93940, USA.

Cook, R. J. (1984): Root Health Importance and Relationship to Farming Practices. In:"Organic Farming Current Technology and Its Role in a Sustainable Agriculture", D.F. Bezdicek and J.F. Power (Ed.) pp. 111-127, ASA Spec. Publ., 46, ASA, CSSA, SSSA, Madison, WS. 
Dogan, R. and U. Bilgili (2010): Effects of previous crop and $\mathrm{N}$-fertilization on seed yield of winter wheat (Triticum aestivum L.) under rain-fed Mediterranean conditions. Bulg. J. Agric. Sci., 16 (6): 733-739.

Ercoli, L.; A. Masonib; S. Pampanab; M. Mariottic and I. Arduinib (2013): As durum wheat productivity is affected by nitrogen fertilisation management in Central Italy. Europ. J. Agron., 44: 38-45.

El-Temsah, M. E. (2017): Response of wheat yield, its components and technological characteristics to different nitrogen rates and planting methods. Egypt. J. Agron., 39 (3): 421- 429.

El-Agrodi, M. W.; A. M. EL-Ghamry and H. H. Ibrahim (2011): Effect of nitrogen fertilizer rates, timing and splitting application on wheat plant grown on reclaimed soils. J. Soil Sci. and Agric. Eng., Mansoura Univ., 2 (9): 915-924.

Gomez, K. A. and A. A. Gomez (1984): Statistical Procedures for Agricultural Research. $2^{\text {nd }} \mathrm{ed.} \mathrm{John}$ Willey and Sons, New York. 97-411.

Haque, A. N. A.; M. E. Hossain; M. E. Haque; M. M. Hasan; M. A. Malek; M. Y. Rafii and S. M. Shamsuzzaman (2017): Response of yield, nitrogen use efficiency and grain protein content of wheat (Triticum aestivum L.) varieties to different nitrogen levels. Bangladesh J. Bot., 46 (1): 389-396.

Haile, D.; D. Nigusse and A. Ayana (2012): Nitrogen use efficiency of bread wheat: Effects of nitrogen rate and time of application. J. Soil Sci. and Plant Nutr., 12 (3): 389-409.

Hefny, Y. A. (2012): Effect of crop sequence and mineral, organic and bio nitrogen fertilization on productivity of wheat. Ph. D. Thesis, Fac. Agric., Cairo, AlAzhar Univ., Egypt.
Kaur, S.; N. Kaur; K. H. M. Siddique and H. Nayyar (2016): Beneficial elements for agricultural crops and their functional relevance in defence against stresses. Arch. Agron. Soil Sci., 62: 905-920.

Mansour, H. A.; S. Pibars; M. S. Gaballah and K. A. S. Mohammed (2016): Effect of different nitrogen fertilizer levels, and wheat cultivars on yield and its components under sprinkler irrigation system management in sandy soil. Internat. J. ChemTech Res., 9 (9): 1-9.

Mandic, V.; V. Krnjaja; Z. Tomic; Z. Bijelic; A. Simic; D. R. Muslic and M. Gogic (2015): Nitrogen fertilizer influence on wheat yield and use efficiency under different environmental conditions. Chilean J. Agric. Res., 75 (1): 92-97.

Rahimizadeh, M.; A. Kashani; A. Zare-Feizabadi; A. R. Koocheki and M. Nassiri-Mahallati (2010): Nitrogen use efficiency of wheat as affected by preceding crop, application rate of nitrogen and crop residues. Australian J. Crop Sci., 4 (5):363-368.

Said, M. T. (2007): Effect of different systems of intensification and fertilization on some wheat cultivars. M. Sc. Thesis, Fac. Agric., Assiut Univ., Egypt.

Singh, R. K.; P. Kumar; B. Prasad; A. K. Das and S. B. Singh (2016): Effect of split application of nitrogen on performance of wheat (Triticum aestivum L.). Internat. J. Agric. Sci., 12 (1): 32-37.

USDA (2018): htpp://apps.fsa. usda.gov/psolonline/ circulars/production.

Usman, K. and A. Ur-Rehman (2014): Response of wheat to tillage and nitrogen application in a cotton-based cropping system. Sarhad J. Agric., 30 (4): 386-392.

Zaheer, S.; A. Ahmed; A. Jan; K. Akhtar and V. N. Ha (2015): Effect of nitrogen and preceding cropping pattern on yield and yield components of rainfed wheat. J. Environ. and Earth Sci., 5 (11): 47-55.

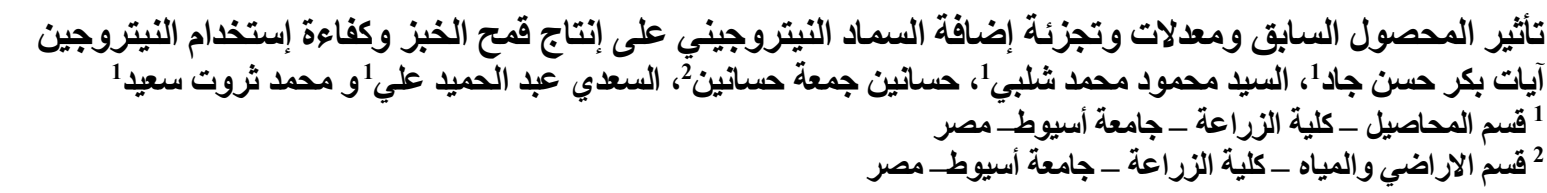

أجريت تجربة حقلية بمزر عة قسم المحاصيل, كلية الزر اعة بجامعة أسبوط خلال موسمي 2016/2015 , 2017/2016 وذللك لدر اسة

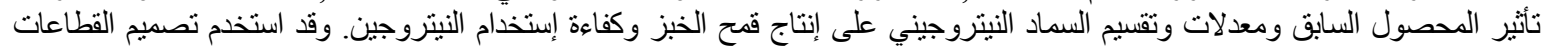

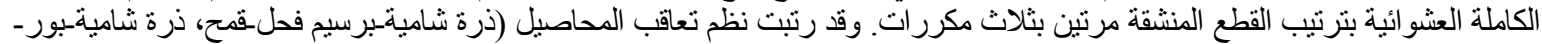

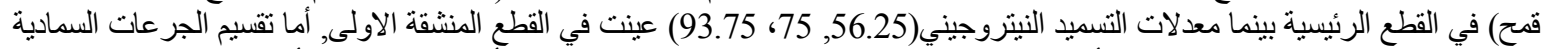

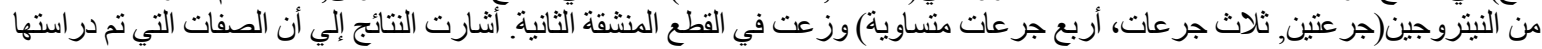

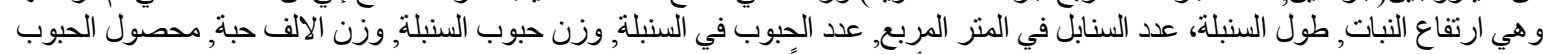

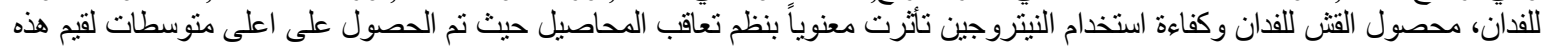

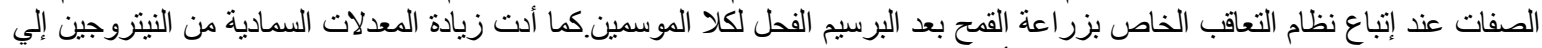

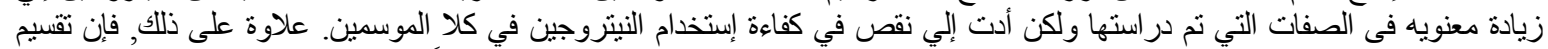

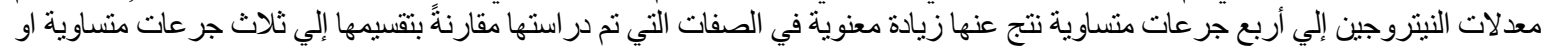

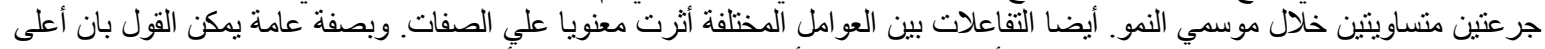

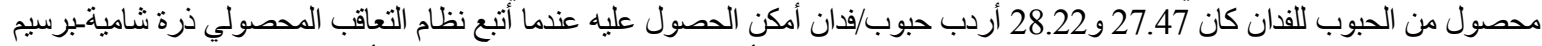
فحلققح وتم التسميد بمعدل 93.75 كجم نبتروجين/فدان يتم إضافتها على أربع جر عات منساوية في الموسم الأول و الثاني على التو الي. 